\title{
Genetic and histological subtypes of gastric cancer reviewed, particularly emphasising on microsatellite instability and E-cadherin gene mutation
}

\author{
Katarzyna Karpińska, Magdalena Lewandowska, Elżbieta Urasińska
}

Almost one million new cases of gastric cancer (GC) were estimated globally in 2012, (i.e. 952,000, representing 6.8\% of the total cancer burden), making it the fifth most common malignancy in the world. GC represents a biologically and genetically diverse group of tumours with multifactorial aetiologies; both environmental and genetic. The vast majority of GCs are adenocarcinomas, which can be further subdivided into intestinal and diffuse histological subtypes according to the Lauren classification published in 1965. The molecular classification of GC according to the Cancer Genome Atlas (TCGA) divides GC into four subtypes: tumours positive for the EBV virus (9\%), microsatellite unstable tumours (22\%), genomically stable tumours (20\%) and tumours with chromosomal instability (CIN) at $50 \%$. Most GCs are sporadic by nature, where approximately $10 \%$ appear to possess a familial predisposition of which around half can be attributed to hereditary germline mutations i.e. those of the E-cadherin (CDH1) or mismatch repair (MMR) genes. Histopathological characteristics of the tumour type and analysis of potential genetic changes have substantial clinical significance, as they determine the choice of treatment. In this review, we consider the molecular pathogenesis, phenotype and testing of GC placing particular emphasis on microsatellite instability (MSI) and the CDH1 mutation.

NOWOTWORY J Oncol 2017; 67, 3: 193-200

Key words: gastric cancer, histopathological classification, microsatellite instability, E-cadherin gene mutation

\section{Introduction}

GC represents a biologically and genetically heterogeneous group of tumours arising from multifactorial aetiologies; both environmental and genetic. Nearly one million new cases of gastric cancer (GC) were estimated globally in 2012 (952,000, i.e. constituting 6.8\% the whole cancer burden), making it the fifth most common malignancy in the world after cancers of the lung, breast, colorectum and prostate. This represents a substantive change since the very first estimates from 1975, when GC was the most common neoplasm. More than $70 \%$ of cases $(677,000)$ occur in developing countries, $(456,000$ in men and 221,000 women), with half of the world's total coming from Eastern Asia; mainly China [1]. The age standardised incidence rate for males is generally twice that for females [2] and the majority of GCs arise in the distal stomach (non-cardia gastric cancers) with their incidence gradually decreasing throughout the world [2]. Cancers of the gastric cardia are most commonly reported in European and North American populations with a rising prevalence [2]. GC is clinically classified as early or advanced stage to help determine prognosis and appropriate treatment. Additionally, histopathological characteristics of the tumour type and analysis of potential genetic disorders have substantial clinical significance, as they ultimately determine the choice of treatment, i.e. submucosal endoscopic dissection which is used as a treatment for early gastric cancer that fulfil the expanded criteria [3].

In this review, we consider the molecular pathogenesis, phenotype and testing of GC, laying particular emphasis on microsatellite instability (MSI) and E-cadherin mutations.

\section{Molecular classification of gastric cancer}

GCs are solid tumours with complex genetic and environmental interactions that contribute towards their initiation 
and progression [4]. Most of GCs are sporadic tumours where genetic alterations are frequently reported. Nevertheless, no major high-penetrance genes have yet been discovered [5], although genetic factors might play an important role in gastric carcinogenesis by possibly affecting immune and inflammatory responses, especially in cases of the bacterium Helicobacter pylori (H. pylori) and Epstein-Barr virus (EBV) infections and thereby altering the susceptibility to gastric cancer [6-8]. GCs classified molecularly by the Cancer Genome Atlas (TCGA) categorises GC into four subtypes: tumours positive for the EBV virus (9\%), tumours with microsatellite instability (22\%), genomically stable tumours (20\%) and tumours with chromosomal instability (CIN) at 50\% [9]. All EBV-positive tumours displayed CDKN2A (p16INK4A) promoter hypermethylation, but lacked the $M L H 1$ hypermethylation characteristic of the MSI-associated CpG island methylator phenotype (CIMP) [9]. EBV-positivity decreased with age among men; more steeply for those tumours localised in the antrum [5]. In addition, EBV-positivity has been associated with longer survival, suggesting that EBV-positive GC may have distinct clinical and genetic features, and therefore may be a separate clinical entity [5]. Sporadic GCs with microsatellite instability present epigenetic silencing of $M L H 1$ in the context of a CIMP, whereas a small minority of GC cases are associated with germline mutation of mismatch repair genes $(M M R)$. The term CIN refers to an accelerated rate of gain and loss of whole or a large portion of chromosomes. The consequence of CIN is an imbalance in chromosomal number (reflected by aneuploidy) and higher rates of losing heterozygosity ( $\mathrm{LOH})$, gene deletions and/or amplifications $[10,11]$. All these alterations may lead to oncogene activation and/or tumour suppressor gene inactivation [11]. Each subtype was found throughout the stomach, but CIN tumours rates were higher in the gastroesophageal junction/cardia, whereas most EBV-positive tumours occurred in the gastric fundus or body [9]. Genomically stable tumours were diagnosed at an earlier age, whereas MSI tumours were diagnosed at relatively older ages [7]. MSI patients tended to be female, but most EBV-positive GCs were observed in males [2]. Approximately $10 \%$ of GCs appear to have a familial predisposition, of which about half can be attributed to hereditary germline mutations as follows: hereditary diffuse gastric cancer with E-cadherin $(\mathrm{CDH} 1)$ mutation, gastric adenocarcinoma and proximal polyposis of the stomach (implicated genes are unknown), hereditary nonpolyposis colorectal cancer (HNPCC) with mismatch repair (MMR) gene mutations, Li-Fraumeni syndrome with TP53 mutation, Peutz-Jeghers syndrome with STK11 gene mutation and Familial adenomatous polyposis with $A P C$ gene mutation $[4,9,12-14]$.

\section{Histopathological subtypes of gastric cancer}

The vast majority of GCs are adenocarcinomas, which can be further subdivided into intestinal and diffuse sub- types according to the Lauren classification published in 1965 [4, 7, 15] (Fig. 1). These subtypes have different molecular profiles and their developmental pathways are distinct $[4,15]$. The relative proportions of GC subtypes worldwide are $74 \%$ intestinal ones versus $16 \%$ diffuse and $10 \%$ other, although diffuse GC is becoming relatively more common in Western countries $[12,16]$. Geographic variations in GC rates correlate with lifestyle, diet and $H$. pylori infection $[5,12]$. The effects of environmental factors as causes of GC is highlighted by declining rates of intestinal GC in immigrants from high-incident countries to low-incident countries $[10,17,18]$. The intestinal subtype is more often seen in older patients, whereas the diffuse type affects younger patients and has a more aggressive clinical course [12]. The intestinal type gastric tumours often present solid masses and arise from a premalignant gastric lesion, such as chronic atrophic gastritis, intestinal metaplasia and dysplasia, that in turn develops into a chronic inflammatory background that is usually induced by H. pylori infection [4, 12]. The 'Correa Model' describes a multistep cascading process from chronic gastritis to atrophic gastritis, intestinal metaplasia and dysplasia before culminating in intestinal-type GC [19]. GCs with chromosomal instability are recognised by intestinal histology [9]. Abrogation of normal p53 function appears to be important in intestinal-type GC and its premalignant counterparts [12]. A genomically stable group of GCs revealed enriched diffuse histological subtypes [19]. Sporadic diffuse GC appears not to develop in this step-wise neoplastic progression, arising instead from normal gastric mucosa with no definitive premalignant stage and is often associated with negative $H$. pylori status [4]. Diffuse tumours exhibit isolated cells, typically developing below the mucosal lining, often spreading and thickening until the stomach appears hardened into a morphological designation termed 'linitis plastica'. Diffuse gastric tumours frequently present as'signet ring cells', named for the marginalisation of the nucleus to the cell periphery due to high mucin content $[12,15]$. The importance of distinguishing between these two main histopathological-types of GC, one with a diffuse component (isolated cells and mixed types) and one without a diffuse component (glandular/intestinal and solid types), is highlighted by finding somatic $E$-cadherin mutations exclusively in the first group [13].

\section{Microsatellite instability in gastric cancer}

Over 130 genes are thought to be involved in DNA repair mechanisms in humans. One of the DNA repair mechanisms is MMR, which includes single strand DNA damage-microsatellite instability (MSI). MSIs are insertion and deletion mutations at microsatellites, because these structures are particularly prone to DNA replication. Microsatellites are defined as stretches of DNA sequences where a single nucleotide (mononucleotides) or units of two or more nucleotides 

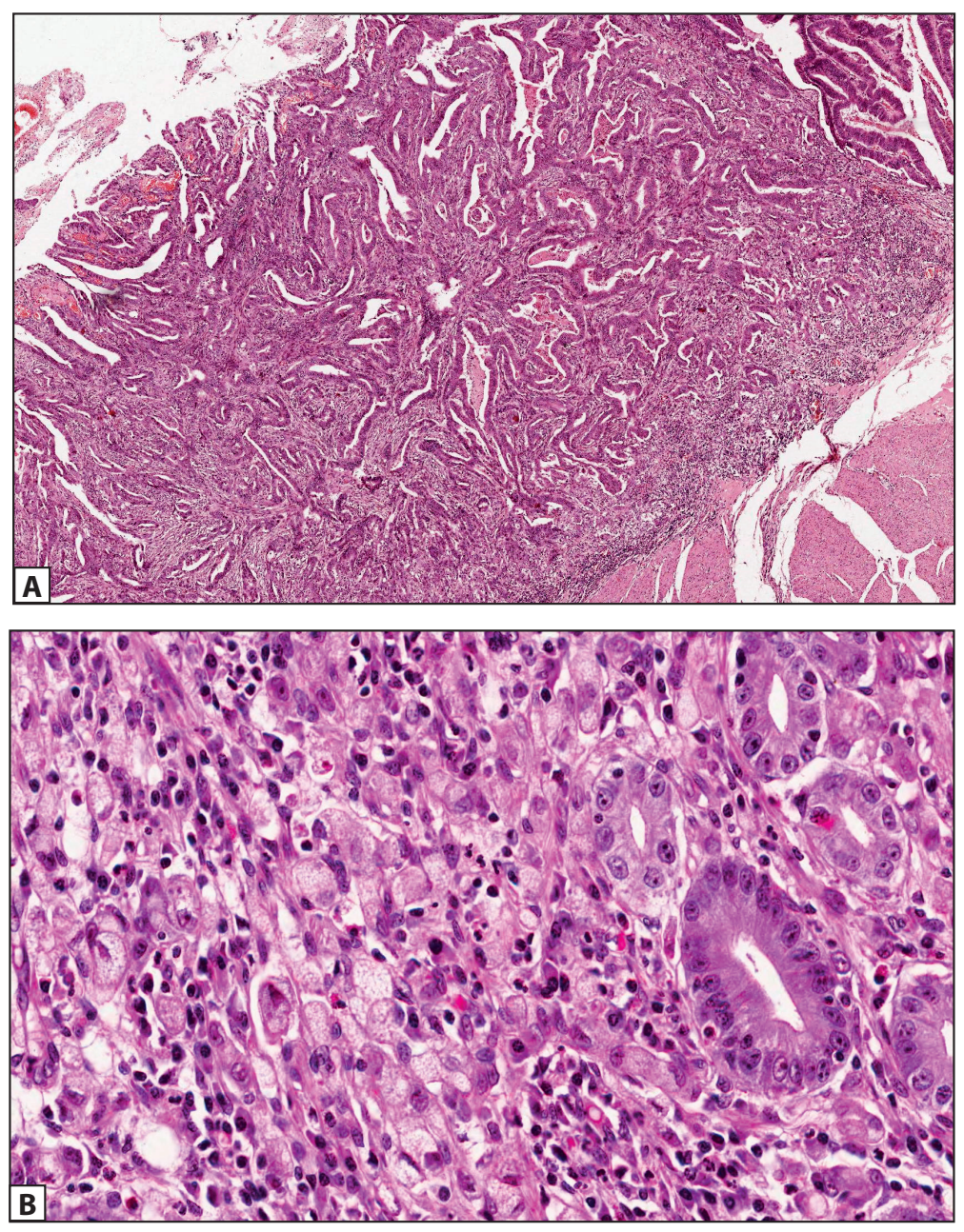

Figure 1. Histological types of GC according to the Lauren classification in H\&E staining. A - intestinal type of GC with tubular arrangement; B - diffuse type of GC with signet ring cell morphology and remnants of benign glands

are repeated in the genome [22]. There are at least 500,000 microsatellites in the human genome, either at intergenic or noncoding regions (with unknown functional significance) as well as in gene-encoding regions (coding microsatellites, cMSs). They are commonly located in introns, with many microsatellites found in promoters, untranslated terminal regions and indeed in coding exons. Insertions and deletions in CMSs result in the production of a truncated and therefore inactive protein. Examples of genes containing coding repeats that are targets for mutations in cancers with MSI, include genes associated with DNA repair $(M L H 1$, MSH2, MSH6, PMS2), apoptosis (BAX, BCL10, Caspase-5), signal transduction (TGF- $\beta$ RII, ACT RII) , cell cycle (PTEN, RIZ), and transcription factors (TCF-4) [22-24].

MMR proteins form heterodimers when active. MLH1 constructs a functional complex with PMS2 and MSH2 along with partner MSH6 [10]. It is of note that the MLH1 and MSH2 proteins are obligatory partners of their retrospective heterodimers [10]. Mutations in the $M L H 1$ or $M S H 2$ gene result in proteolytic degradation of the retrospective dimer and consequent loss of both the obligatory and the secondary partner proteins [10]. The reverse, however, is not true: a mutation in one of the secondary genes, i.e. PMS2 or MSH6, does not usually lead to a concurrent loss of the obligatory proteins (MLH1 or MSH2, respectively) [10].

The MMR system is necessary for maintaining genomic stability by correcting single-base mismatches as insertion-deletion loops which form during DNA replication [25]. Impaired MMR function leads to high-level MSI, which may be observed in both sporadic and hereditary cancers [10,22]. The MSI-H phenotype is reported in $5-50 \%$ of all GCs with significant differences within various ethnic groups [26, 27]. The overwhelming majority of these are sporadic neoplasms with hypermethylation of $\mathrm{CpG}$-rich gene promoters, thereby leading to transcriptional inactivation of a large number of genes, including MMR genes [11, 22]. MicroRNAs have been shown to act as oncogenes or tumour suppressing genes in cancer, with putative growth-inhibitory functions undergoing promoter CpG island hypermethylation in human cancer [22]. Recognising the link between miR-155 
Table I. Amsterdam Criteria I and Amsterdam Criteria II for diagnosing Lynch Syndrome [1-20]

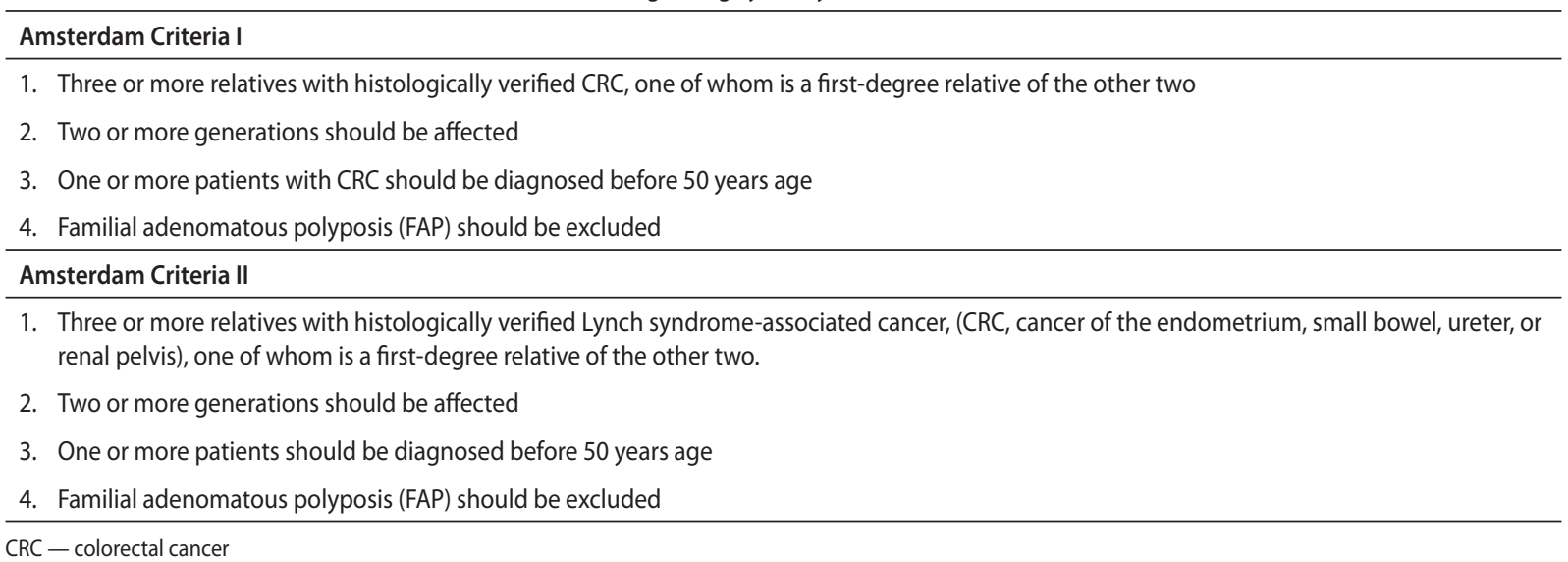

with oncogenic function has been demonstrated through their specific downregulation of $M L H 1, M L H 2$ and MSH6 (core components of DNA mismatch repair), which has been implicated in the pathogenesis of HNPCC [22]. In particular, an altered expression of MLH1 has been associated with gene inactivation by promoter hypermethylation [11]. The silencing of these genes is responsible for MSI development $[10,22]$. Cancers with a high degree of methylation (CIMP phenotype) represent a clinically and aetiologically distinct group of sporadic GCs characterised by epigenetic instability [10, 12]. MSI-H GCs develop and progress genetically as a consequence of frameshift mutations in multiple target genes [26]. TGF $\beta R / /$ mutations have appeared to play a major role in gastric tumourigenesis, thus suggesting that progression in MSI-H GCs is linked to cellular escape from the growth control signal of the TGF- $\beta$ network [26].

A germline mutation in one of the $M M R$ genes is responsible for genetic predisposition to many cancers known as the Lynch syndrome, previously referred to as HNPCC [9, 22]. The Lynch syndrome is an autosomal dominant disorder with high penetrance that accounts for $5 \%$ of newly diagnosed cases of GC $[12,22]$. Patients with Lynch syndrome also exhibited an increased risk of GC and other extracolonic malignancies [10, 22]. The life time risk of GC for patients with Lynch syndrome is 6 to $13 \%$ [12]. The median age at diagnosis is 71 years for all GC types and 56 years for patients with Lynch syndrome [12]. From a study with patients belonging to families diagnosed with Lynch syndrome, more than
$80 \%$ of tumours display MSI [22]. The most common cause of MSI being absent in Lynch syndrome is a false negative result, because of inadequate numbers of microsatellite markers or an inadequate proportion of tumour cells in the sample [22].

In Amsterdam, from 1990, the first set of clinical criteria for families with Lynch syndrome was established [28]. The expanded criteria include also extracolonic tumour sites as diagnostic features (Tab. I) [28-30]. The Amsterdam Criteria were initially designed to serve research purposes. The Bethesda Guidelines and revised Bethesda Guidelines are used to select patients for MSI testing, i.e. to limit molecular analysis to cancers that have a high likelihood of being hereditary (Tab. II) [30, 31]. A standard test for MSI, also known as the Bethesda panel, was proposed at the National Cancer Institute in 1997 but also customized panels are used which precisely indicate MSI $[24,32,33]$. This is a panel consisting of two mononucleotide repeats (BAT25, BAT26) and three dinucleotide repeats (D2S123, D5S346, D17S250) [22]. Instability can thereby be classified in high-level MSI (MSI-H) with instability demonstrated by the five Bethesda panels and low-level MSI (MSI-L) with instability at only one of the five Bethesda panels [22]. Microsatellite stable (MSS) GC presents as a non-positive marker by the Bethesda panel [34]. Suraweera et al. proposed a new five-marker or pentaplex panel for MSI screening that comprises the mononucleotide repeats BAT25, BAT26, NR21, NR22 and NR24. The pentaplex assay is commercially available and has been used for se-

Table II. Revised Bethesda Guidelines [20, 21]. Colorectal cancers (CRCs) should be tested for MSI in the following cases:

\footnotetext{
1. CRC diagnosed in patients below 50 years age

2. Presence of synchronous or metachronous CRC or Lynch syndrome-associated tumour*, regardless of age

3. CRC with MSI-H histology diagnosed in patients below 60 years age

4. Patients with CRC and CRC or a Lynch syndrome-associated tumour* diagnosed in at least one first-degree relative aged below 50 years

5. Patients with CRC and CRC or a Lynch syndrome-associated tumour* diagnosed in two first-degree or second-degree relatives regardless of age
}

*Lynch syndrome-associated tumours include cancers of the colorectum, endometrium, stomach, ovary, pancreas, biliary tract, small bowel, ureter, renal pelvis and brain tumours (usually glioblastoma as seen in the Turcot syndrome), as well as sebaceous gland adenomas and keratoacanthomas (in the Muir-Torre syndrome); CRC - colorectal cancer; MSI-H - microsatellite instability high 

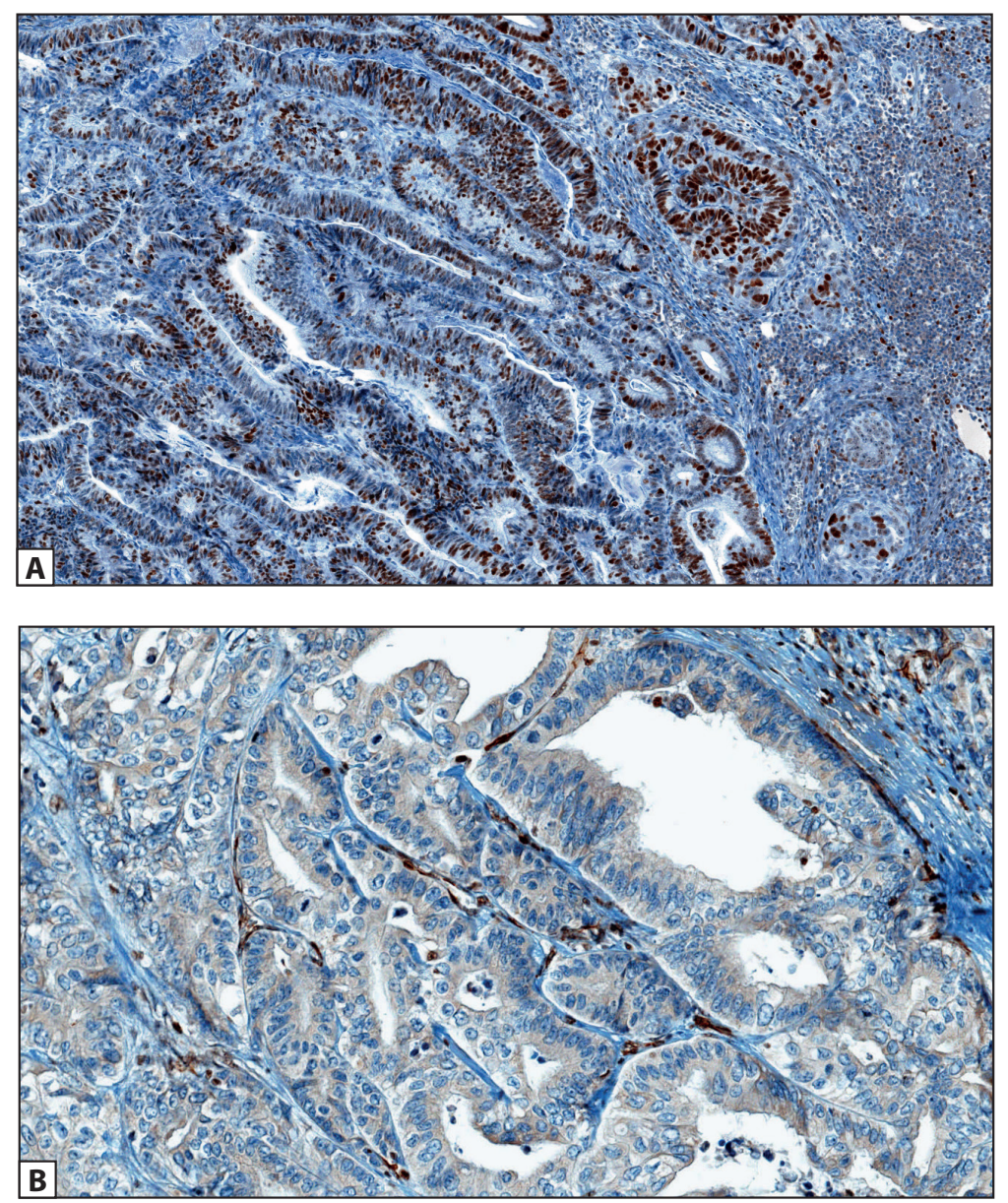

Figure 2. Immunohistochemical staining for MSH6 and MSH2 proteins in GC. A - malignant tubules of GC and benign lymphoid cells showing positive nuclear staining for MSH6 protein; B - MSI-H AGC exhibiting a complete loss of MSH2 expression, with stromal cells showing positive staining

veral years, although the simultaneous assessment of two markers (BAT26 and NR24) has been shown to be as effective as the pentaplex panel for diagnosing MSI [22]. The MMR defect may also be screened with the immunohistochemistry method; MMR protein expression. Recent studies have demonstrated the predictive value of immunohistochemistry which includes analysing four proteins (MLH1, MSH2, MSH6 and PMS2) which is virtually equivalent to MSI testing (Fig. 2) [11, 35, 36]. Microsatellite analysis and immunohistochemical staining are in agreement in more than $90 \%$ of GCs [37]. However, microsatellite analysis when using PCR is more sensitive and provides informative results in virtually all cancers [37].

Clinicopathological features displayed by high-level microsatellite instability GC with MSI-H are reported to be distinct both clinically and in molecular terms compared to microsatellite stable (MSS) GC [35]. In contrast to colorectal cancer, the clinical significance and role of MSI in GC however remain controversial [35]. MSI-H GCs tend to be associated with an older age, female gender, distal tumour location, rare lymph node involvement and a lesser propensity to invade the serosa. They often display distinct features at the histological level, which should raise suspicion of MSI and prompt further analysis [35]. The histological features which are not specific, but are commonly seen, include an intestinal type of tumour and prominent lymphoid cell infiltration with expanding borders $[26,37,38]$. The MSI-H phenotype was also associated with mucinous GC or MUC 6 positivity $[26,35]$. There exists a growing body of evidence that GC with MSI is associated with a better prognosis than MSS GC $[39,40]$.

Most histological features which serve as diagnostically useful markers of MSI-H status are apparent in both sporadic and hereditary GC in the Lynch syndrome.

\section{E-cadherin mutation and gastric cancer}

The E-cadherin gene coding sequence gives rise to a mature protein consisting of 3 major domains, a large extracellular domain and a smaller transmembrane and cytoplasmic domain $[12,41]$. As in other autosomal dominant 
cancer predisposing genes, only $1 \mathrm{CDH} 1$ allele is mutated in the germline and the majority of genetic changes lead to truncation of the protein. $\mathrm{CDH} 1$ encodes the calcium-dependent cell-adhesion glycoprotein E-cadherin that connects to the actin cytoskeleton through a complex with catenin proteins (Fig. 3) [41-43]. Functionally, E-cadherin plays a role in maintaining normal tissue morphology and cellular differentiation [44]. $\mathrm{CDH} 1$ somatic mutations have been noted in genomically stable subtypes (37\% of cases) [9]. The germline $\mathrm{CDH} 1$ mutation has been found to be associated with approximately $30 \%$ of families with hereditary diffuse gastric cancer (HDGC), with a lifetime risk of GC greater than $80 \%$, and up to a $60 \%$ risk for female carriers developing lobular breast cancer $[27,45]$. The average age of onset for both males and females is 38 years, though cases of onset at ages 15 and 16 years have been documented worldwide [13]. $C D H 1$ is the only gene implicated in HDGC. About $100 \mathrm{CDH} 1$ mutation-positive families have been globally reported [6]. Using the initial International Gastric Cancer Linkage Consortium (IGCLC) criteria for HDGC (Tab. III), CDH1 mutation testing yielded a detection rate of 30-50\% [46] of clinically diagnosed HDGC families with no identifiable genetic mutation [12]. A somatic $C D H 1$ mutation was identified in about half of sporadic diffuse GCs, but occurs rarely for intestinal GC [12]. The histological phenotype of HDGC at the early stage includes patchy intramucosal signet ring carcinoma cells in the lamina propria which is a unique feature of carcinoma in situ associated with the pagetoid spread of tumour cells along the basement membrane [14]. Although malignant foci are generally localised to the proximal one third of the stomach, lesions may be distributed through the entire stomach [47]. GC in patients with HDGC syndrome presents a diffuse pattern of growth with a high intracellular mucin content, signet ring features and a loss of E-cadherin upon immunohistochemical analysis (Tab. III). Prophylactic gastrectomy is offered to carriers of the germline $\mathrm{CDH} 1$ mutation [12]. Nearly all specimens contain multiple foci of intramucosal diffuse signet ring cell carcinoma [12].

\section{Conclusions}

Diagnosing gastric cancer usually begins by histological examination of material from a gastric biopsy. Clinical manifestations, (a family history, the presence of potential risk factors of GC, patient age, a macroscopic tumour image), and the histological type of cancer may help to determine those patients who might need future treatment because of their genetic predisposition to GC. Finally, as aforementioned in detail, the two principal subtypes of GC with MSI-H and $\mathrm{CDH} 1$ mutation evolve through different pathways, and those differences in molecular pathogenesis translate into a morphological distinction, which merits our attention $[48,49]$.

Identifying MSI-H and $\mathrm{CDH} 1$ mutated $\mathrm{GC}$ is of considerable clinical significance. The MSI-H status is not only the cen-

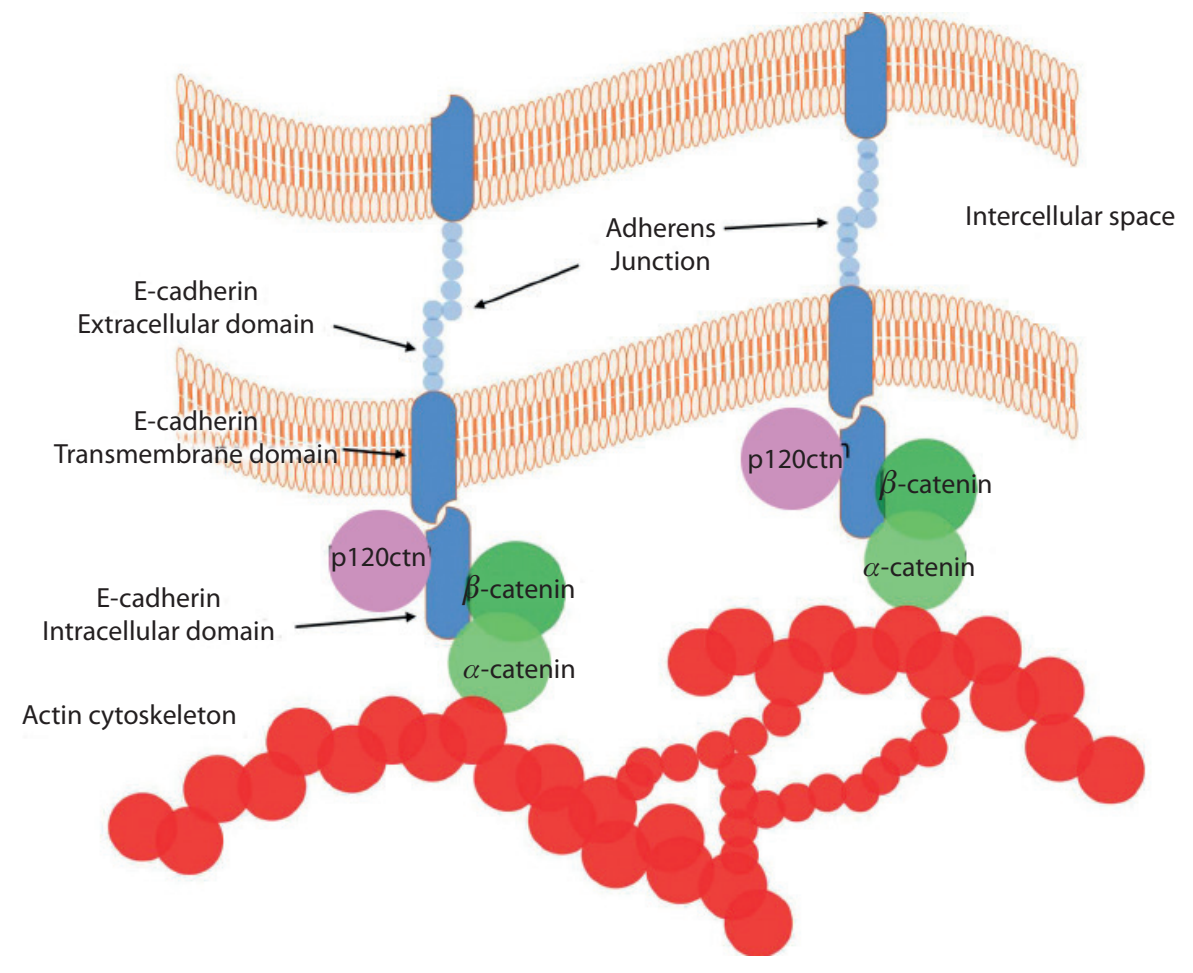

Figure 3. E-cadherin interface mediating cell-cell adhesion. The extracellular domain binds to the extracellular domain of E-cadherin on adjacent cells by calcium activated dimerisation. The intracellular domain binds to p120ctn and to $\beta$-catenin. The complex formed with $\beta$-catenin allows $\alpha$-catenin to link this complex to the cellular actin cytoskeleton [39] 
Table III. Clinical criteria for CDH1 testing defined by IGCLC 2010 [33]

\begin{tabular}{l}
\hline Clinical criteria for CDH1 testing defined by IGCLC 2010 \\
\hline 1. Two GC cases in the family: one confirmed diffuse type, one diagnosed at age $<50$ years \\
2. Three confirmed diffuse GC in first-or second-degree relatives, independent of age \\
3. Diffuse GC diagnosed at age $<40$ years (no additional family history needed) \\
4. Personal or family history (first-or second-degree) of diffuse GC and lobular breast cancer, with one diagnosed at age $<50$ years \\
\hline
\end{tabular}

GC — gastric cancer; IGCLC — The International Gastric Cancer Linkage Consortium

tral molecular tumour feature for identifying individuals with Lynch syndrome and sporadic MMR deficient GCs, but it is also a marker of favourable outcome and a predictive marker of resistance to the standard 5-fluorouracil-based adjuvant chemotherapy $[38,50]$. Patients with the $\mathrm{CDH} 1$ mutation require individual clinical therapy, (preventive gastrectomy may be considered and endoscopic submucosal dissection can be used as an alternative method of treatment), and regular endoscopic examination using chromoendoscopy for direct biopsy for diagnosing early diffuse GC.

\section{Conflict of interest: none declared}

\section{Katarzyna Karpińska, MD, PhD}

Department of Pathology, Pomeranian Medical University Unii Lubelskiej $1 \mathrm{St}$.

71-252 Szczecin, Poland

e-mail:kkarp@pum.edu.pl

Received: 26 Jan 2017

Accepted: 16 May 2017

\section{References}

1. Ferlay J, Soerjomataram I, Ervik M et al. GLOBOCAN 2012 v1.0. Cancer incidence and mortality worldwide: IARC Cancer Base No.11, Lyon: International Agency for Research on Cancer, 2013. Available from: http://globocan.iarc.fr, accessed on day/month/year.

2. Ang TL, Fock KM. Clinical epidemiology of gastric cancer. Singapore Med J 2014; 55: 621-628.

3. Japanese Gastric Cancer Association. Japanese gastric cancer treatment guidelines 2010 (ver.3). Gastric Cancer 2011; 14: 113-123.

4. MacLean MH, El-Omar EM. Genetics of gastric cancer. Nature Rev Gastroenterol Hepatol 2014; 11: 664-674.

5. Park JY, von Karsa L, Herrero R. Prevention strategies for gastric cancer: A global perspective. Clin Endosc 2014; 47: 478-489.

6. Guilford P, Humar B, Blair V. Hereditary diffuse gastric cancer: translation of $\mathrm{CDH} 1$ germline mutation into practice. Gastric Cancer 2010; 13: 1-10.

7. Jacome AA, Coutinho AK, Lima EM et al. Personalized medicine in gastric cancer: where are we and where are we going? World $J$ Gastroenterol 2016; 22: 1160-1171.

8. Tarazona N, Gambardella V, Huerta M et al. Personalised treatment in gastric cancer: myth or reality? Curr Oncol Rep 2016; 18: 41.

9. Cancer Genome Atlas Research Network. Comprehensive molecular characterisation of gastric adenocarcinoma. Nature 2014; 513: 202-209.

10. Setaffy $L$, Langner C. Microsatellite instability in colorectal cancer: clinicopathological significance. Pol J Pathol 2015; 66: 203-218.

11. Nobilli S, Bruno L, Landini I et al. Genomic and genetic alteration influence the progression of gastric cancer. World J Gastroenero/ 2011;17:290-299.

12. Chun N, Ford JM. Genetic testing by cancer site: stomach. Cancer J 2012; 18: 355-363.

13. Caldas C, Carneiro F, Lynch HT et al. Familial gastric cancer: overview and guidelines for management. J Med Genet 1999; 36: 873-880.

14. Hu B, Hajj N, Sittler S et al. Gastric cancer: Classification, histology and application of molecular pathology. J Gastrointest Oncol 2012; 3: $251-261$.
15. Lauren P. The two histological main types of gastric carcinoma: diffuse and so-called intestinal-type carcinoma. Acta Path Microbiol Scand 1965; 64: 31-49.

16. Wu H, Rusiecki JA, Zhu K et al. Stomach carcinoma incidence patterns in the United States by histologic type and anatomic site. Cancer Epidemiol Biomarkers Prev 2009; 18: 1945-1952.

17. Sipponen P. Biomarkers in clinical practice: a tool to find subjects at high risk for stomach cancer. A personal view. Adv Med Sci 2006; 51: 51-53.

18. Leja $M$, Kupcinskas $L$, Funka $K$ et al. Value of gastrin-17 in detecting antral atrophy. Adv Med Sci 2011; 56 : 145-150.

19. Correa P. Helicobacter pylori and gastric carcinogenesis. Am J Surg Pathol 1995; 19 Suppl 1: S37-S43.

20. Tan IB, Ng I, Tai WM et al. Understanding the genetic basis of gastric cancer: recent advances. Expert Rev Gastroenterol Hepatol 2012; 6: 335-341.

21. Guilford P, Humar B, Blair V. Hereditary diffuse gastric cancer: translation of $\mathrm{CDH} 1$ germline mutations into clinical practice. Gastric Cancer 2010; 13: 1-10.

22. Centelles J. General aspect of colorectal cancer. ISRN Oncol 2012; 2012: 139268.

23. Gryko M, Pryczynicz A, Guzińska-Ustymowicz $K$ et al. Immunohistochemical assessment of apoptosis-associated proteins: p53, Bcl-xL, Bax and Bak in gastric cancer cells in correlation with clinical and pathomorphological factors. Adv Med Sci 2012; 57: 77-83.

24. Boland CR, Thibodeau SN, Hamilton SR et al. A National Cancer Institute Workshop on Microsatellite Instability for cancer detection and familial predisposition: development of international criteria for the determination of microsatellite instability in colorectal cancer. Cancer Res 1998; 58:5248-5257.

25. Jasperson KW, Tuohy TM, Neklason DW et al. Hereditary and familial colon cancer. Gastroenerology 2010; 138: 2044-2058.

26. Falchetti M, Saieva C, Lupi R et al. Gastric cancer with high-level microsatellite instability: target gene mutations, clinicopathologic features, and long-term survival. Human Pathol 2008; 39: 925-932.

27. Lee HJ, Jang YJ, Lee EJ et al. The significance of mismatch repair genes in gastric cancer. $J$ Cancer Res Ther 2013; 9: 80-83.

28. Vasen HF, Mecklin JP, Khan PM et al. The International Collaborative Group on Hereditary Non-Polyposis Colorectal Cancer (ICG-HNPCC). Dis Colon Rectum 1991; 34: 424-425.

29. Vasen HF, Watson P, Mecklin JP et al. New clinical criteria for hereditary nonpolyposis colorectal cancer (HNPCC, Lynch syndrome) proposed by the International Collaborative group on HNPCC. Gastroenterology 1999; 116: 1453-1456.

30. Giardiello FM, Allen Jl, Axilbund JE et al. Guidelines on genetic evaluation and management of Lynch syndrome: a consensus statement by the U.S. Multi-Society Task Force on Colorectal Cancer. Gastrointest Endosc 2014; 80: 197-220.

31. Umar A, Boland CR, Terdiman JP et al. Revised Bethesda Guidelines for hereditary nonpolyposis colorectal cancer (Lynch syndrome) and microsatellite instability. J Nat Cancer Inst 2004; 96: 261-268.

32. Musulen E, Sanz C, Munoz-Marmol AM et al. Mismatch repair protein immunohistochemistry: a useful population screening strategy for Lynch syndrome. Human Pathol 2014; 45: 1388-1396.

33. Park J, Shin S, Yoo HM et al. Evaluation of the three customized MS panels to improve the detection of microsatellite instability in gastric cancer. Clin Lab 2017; 63: 705-716.

34. Wu X, Xu Y, Chai W et al. Causal link between microsatellite instability and hMRE11 dysfunction in human cancers. Mol Cancer Res 2011; 9: 1443-1448.

35. Kim JY, Shin NR, Kim A et al. Microsatellite instability status in gastric cancer: a reappraisal of its clinical significance and relationship with mucin phenotypes. Korean J Pathol 2013; 47: 28-35.

36. Gonzalez RS, Messing S, Tu X et al. Immunohistochemistry as a surrogate for molecular subtyping of gastric adenocarcinoma. Hum Pathol 2016; 56: 16-21. 
37. Seo HM, Chang YS, Joo SH et al. Clinicopathologic characteristics and outcomes of gastric cancers with MSI-H phenotype. J Surg Oncol 2009; 99: 143-147.

38. Corso G, Pedrazzini C, Marrelli D et al. Correlation of microsatellite instability at multiple loci with long-term survival in advance gastric carcinoma. Arch Surg 2009; 144: 722-727.

39. Choi YY, Bae J M, An JY et al. Is microsatellite instability a prognostic marker in gastric cancer?: A systematic review with meta-analysis. J Surg Oncol 2014; 110: 129-135.

40. $\mathrm{Kim} \mathrm{H}, \mathrm{An} \mathrm{JY}$, Noh SH et al. High microsatellite instability predicts good prognosis in intestinal-type gastric cancer. $J$ Gastroenterol Hepatol 2011; 26: 585-592.

41. Grunwald GB. The structural and functional analysis of cadherin calcium dependent cell adhesion molecules. Curr Opin Cell Biol 1993; 5: 797-805.

42. Becker KF, Atkinson MJ, Reich U et al. E-cadherin gene mutations provide clues to diffuse type gastric carcinomas. Cancer Res 1994; 54: 3845-3852.

43. Gall TM, Frampton AE. Gene of the month: E cadherin (CDH1). J Clin Pathol 2013; 66: 928-932.
44. Birchmeier W. E-cadherin as a tumour (invasion) suppressor gene. Bioessays 1995; 17: 97-99.

45. Fitzgerald RC, Hardwick R, Huntsman D et al. Hereditary diffuse gastric cancer: updated consensus guidelines for clinical management and directions for future research. J Med Genet 2010; 47: 436-444.

46. Brooks-Wilson AR, Kaurah P, Suriano G et al. Germline E-cadherin mutations in hereditary diffuse gastric cancer: assessment of 42 new families and review of genetic screening criteria. JMed Genet 2004; 41: 508-517.

47. Rogers WM, Dobo E, Norton JA et al. Risk-reducing total gastrectomy for germline mutations in $\mathrm{E}$-cadherin $(\mathrm{CDH} 1)$ : pathologic findings with clinical implications. Am J Surg Pathol 2008; 32: 799-809.

48. Huynh JM, Laukaitis CM. Panel testing reveals nonsense and missense $\mathrm{CDH} 1$ mutations in families without hereditary diffuse gastric cancer. Mol Genet Genomic Med 2016;4: 232-236.

49. Caldeira J, Fiqueiredo J, Brás-Pereira C et al. E-cadherin-defective gastric cancer cells depend on Laminin to survive and invade. Hum Mol Genet 2015; 24: 5891-5900.

50. Hyde A1, Fontaine D, Stuckless S et al. A histology-based model for predicting microsatellite instability in colorectal cancers. Am J Surg Pathol 2010; 3: 93-100. 